Journal of Intelligent Material Systems \& Structures

\title{
Experimental Validation of a Novel Magnetorheological Damper with Internal Pressure Control
}

\begin{tabular}{|c|c|}
\hline Journal: & Journal of Intelligent Material Systems and Structures \\
\hline Manuscript ID & JIM-16-063.R1 \\
\hline Manuscript Types: & SI-2015SMASIS-MSC \\
\hline Date Submitted by the Author: & $\mathrm{n} / \mathrm{a}$ \\
\hline Complete List of Authors: & $\begin{array}{l}\text { Golinelli, Nicola; University of Modena and Reggio Emilia, Dept. of Sciences } \\
\text { and Methods for Engineering } \\
\text { Spaggiari, Andrea; University of Modena and Reggio Emilia, Department of } \\
\text { Science and Methods for Engineering }\end{array}$ \\
\hline Keyword: & $\begin{array}{l}\text { Magnetorheological Damper, design\&manufacturing, experimental } \\
\text { validation }\end{array}$ \\
\hline Abstract: & $\begin{array}{l}\text { In the present paper we investigated the behaviour of magnetorheological } \\
\text { fluids (MRFs) under a hydrostatic pressure up } \\
\text { to } 40 \text { bar. We designed, manufactured and tested a magnetorheological } \\
\text { damper (MRD) with a novel architecture which } \\
\text { provides the control of the internal pressure. The pressure was regulated } \\
\text { by means of an additional apparatus connected to } \\
\text { the damper that acts on the fluid volume. The MRD was tested under } \\
\text { sinusoidal inputs and with several values of magnetic } \\
\text { field and internal pressure. The results show that the new architecture is } \\
\text { able to work without a volume compensator and } \\
\text { bear high pressures. On the one hand, the influence of hydrostatic } \\
\text { pressure on the yield stress of MRFs is not strong probably } \\
\text { because the ferromagnetic particles cannot arrange themselves into thicker } \\
\text { columns. On the other hand, the benefits of the } \\
\text { pressure on the behaviour of the MRD are useful in terms of preventing } \\
\text { cavitation. }\end{array}$ \\
\hline \multicolumn{2}{|c|}{$\begin{array}{l}\text { Note: The following files were submitted by the author for peer review, but cannot be converted to } \\
\text { PDF. You must view these files (e.g. movies) online. }\end{array}$} \\
\hline \multicolumn{2}{|l|}{$\begin{array}{l}\text { reviewed_JIMSS_SMASIS2015.tex } \\
\text { harvard.bst } \\
\text { graphs.rar } \\
\text { imm.rar }\end{array}$} \\
\hline
\end{tabular}




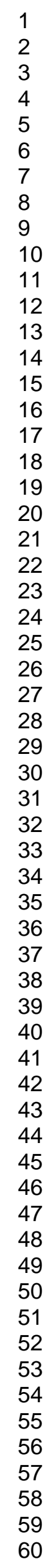

SCHOLARONE $^{\text {It }}$

Manuscripts

http://mc.manuscriptcentral.com/jimss 
Journal of Intelligent Material Systems

\title{
Experimental Validation of a Novel Magnetorheological Damper with Internal Pressure Control
}

\author{
N. Golinelli, A. Spaggiari \\ Department of Sciences and Methods for Engineering, University of Modena and Reggio Emilia, Italy
}

\begin{abstract}
In the present paper we investigated the behaviour of magnetorheological fluids (MRFs) under a hydrostatic pressure up to 40 bar. We designed, manufactured and tested a magnetorheological damper (MRD) with a novel architecture which provides the control of the internal pressure. The pressure was regulated by means of an additional apparatus connected to the damper that acts on the fluid volume. The MRD was tested under sinusoidal inputs and with several values of magnetic field and internal pressure. The results show that the new architecture is able to work without a volume compensator and bear high pressures. On the one hand, the influence of hydrostatic pressure on the yield stress of MRFs is not strong, probably because the ferromagnetic particles cannot arrange themselves into thicker columns. On the other hand, the benefits of the pressure on the behaviour of the MRD are useful in terms of preventing cavitation.
\end{abstract}

Keywords

Magnetorheological damper, design\&manufacturing, experimental validation, squeeze strengthen effect

\section{Introduction}

Magnetorheological fluid dampers are one of the applications that exploit the characteristics of magnetorheological fluids (Carlson and Jolly 2000, Jolly et al. 1999). The purpose of conventional oil-dampers is to dissipate energy by varying the resistance that the fluid encounters when is flowing from the compression chamber to the extension chamber and vice versa. In such devices, the resistance is due to the entity of the chokes through the piston head. The smaller they are the higher is the damping force. MRDs, compared to traditional ones, exploit the change in the rheological behaviour of MRFs in order to achieve controllable damping properties. The change in the rheological properties of magnetorheological fluids occurs when a magnetic field is applied. Such magnetic field is typically produced by a coil wire embedded in the piston head of the damper, in which connecting leads are usually brought out through the hollow piston rod. Nowadays, several MRF dampers have been developed with different architectures (Zhu et al. 2012). Indeed, for every final application that uses these devices from civil to automotive application MRDs' architectures can be adapted to best fit the specifications required. The most common configurations are the mono-tube structure, the twin-tube structure and the double-ended structure. The mono-tube damper is the simpler in terms of mechanical structure. It is based on a single-rod cylinder structure in which a diaphragm (or floating piston) separates the fluid from an accumulator. The accumulator is filled with high pressure

*Corresponding author; e-mail: nicola.golinelli@unimore.it 
gas and is mainly used to accommodate the volume change caused by the rod moving inward the cylinder. The twin-tube structure has an inner and outer cylinder. The inner cylinder is filled with MRF while the external one, that is partially filled with MRF, compensates volume changes as the accumulator in the mono-tube dampers does. A foot valve attached to the bottom of the inner cylinder is employed to regulate the flow between extension and compression chambers. This type of architectures works with lower gas pressure but have more problems in dissipating the generated heat. The double-ended structure is composed of a mono-tube cylinder and two rod attached to the opposite surfaces of the piston head. The piston rods have the same diameter so a rod-volume compensator is no longer required. Hence, as the damper is compressed there is no gas force to push against, making the damping more sensitive. Further studies on the behaviour of MRFs have proved that direct-shear mode and flow mode when coupled with a unidirectional compression can provide higher yield stress (Guo et al. 2013, Tang et al. 2000, Tian et al. 2002a b, 2010, Zhang et al. 2004, Spaggiari and Dragoni 2012, 2013, Becnel and Wereley 2013, Becnel et al.|2015, Mazlan et al. 2008). The phenomenon is known as squeeze strengthening effect. This effect is mainly due to the rearrangement of the micron-sized ferromagnetic particles along the direction of the applied compression. The consequence is the formation of thicker and stronger columns of particles that enhance the yield stress of MRFs. The main aim of the present work concerns the design of a novel damper architecture that would allow the internal pressure regulation. We wanted to investigate the behaviour of magnetorheological fluids under a hydrostatic and uniformly distributed pressure. The changing in the internal pressure was obtained by exploiting the compressibility of MRFs. Indeed, a pressurized system was manufactured in order to control the internal volume of fluid and then its pressure. Several patents pending exists on pressurized magnetorheological dampers (Lau and Liao 2007) (up to 27 bar) and they principally aim at minimizing cavitation in the device. They are mostly based on double-ended (through-rod) structure, in fact, the accumulator chamber is not allowed because it would compensate all the pressure increases. The main difference between the proposed system and the ones from the literature is that we need to avoid air compensation in order to control the pressure, but without a through rod architecture which is not suitable for many applications, due to its moving parts that can be dangerous (i.e. automotive field).

\section{Materials and Methods}

The proposed prototype should permit an active control of the internal pressure and keep its level constant during piston displacement. Hence, the change in the internal volume as well as the stiffening of the damper caused by the piston rod entering into the cylinder has to be avoided. So, the proposed architecture differs from other air-compensated systems since no flexible diaphragms or compressible gases are employed because they would prevent external pressure control as required. Figure 1 shows the conceptual scheme of the damper presented in this paper. We decided to use a counter-rod fixed to the end plug and coupled with the piston head. The counter-rod has the same diameter of the upper-rod so that there is no volume variation. During the piston displacement, the counter-rod moves inward the inner chamber obtained into the piston head. The inner chamber is also directly connected to the canal through the upper-rod in order to bring out the coil wire. Thereby, overpressure or depression within the chamber will not occur. It is worth noting that two coils were adopted. In this way, the longer axial length of the piston head is exploited to maximize the concatenated magnetic flux. The technical specifications of the proposed magnetorheological damper are listed in Table 1

Table 1. Technical specifications.

\begin{tabular}{ll}
\hline Maximum Force $(\mathrm{N})$ & 2000 \\
\hline Maximum Velocity $(\mathrm{mm} / \mathrm{s})$ & 150 \\
\hline Stroke $(\mathrm{mm})$ & 50 \\
\hline Maximum input current $(\mathrm{A})$ & 2 \\
\hline Maximum body diameter $(\mathrm{mm})$ & 50 \\
\hline Maximum pressure (bar) & 40 \\
\hline
\end{tabular}




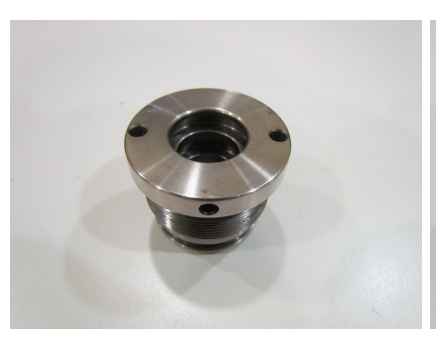

(a)

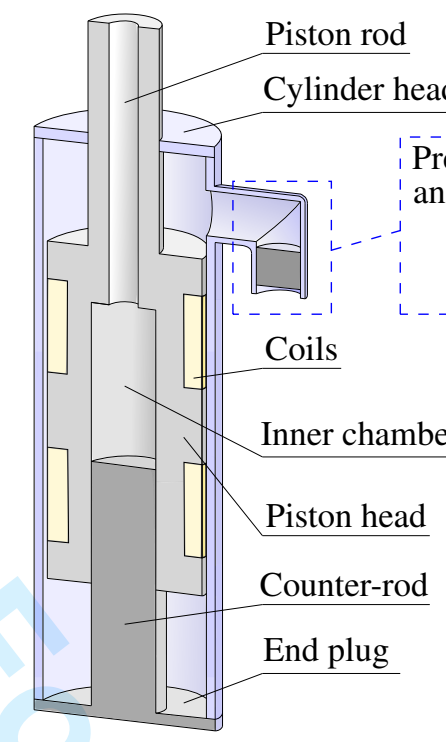

Figure 1. Conceptual scheme of the architecture proposed.

Pressurization system and control cursor

$\Delta V<0$

$\Delta P>0$

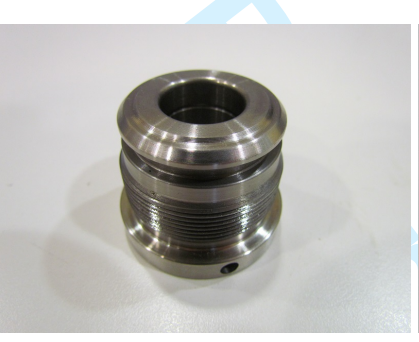

(b)

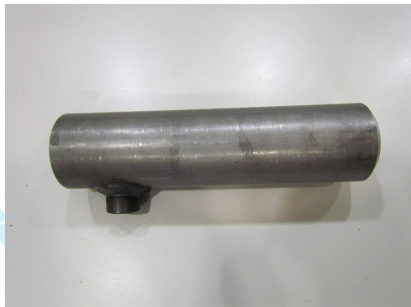

(c)

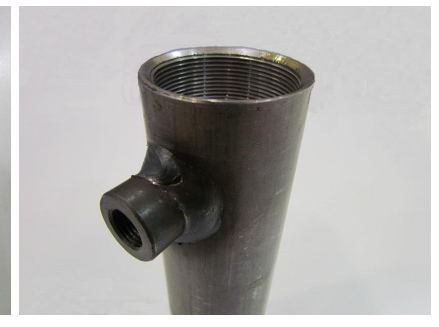

(d)

Figure 2. Commercial components. Cylinder head for a $20 \mathrm{~mm}$ diameter rod (a), (b). Hydraulic cylinder (c) and welded boss for pressurization system (d).

In order to keep the manufacturing of the damper as simple as possible, some commercial components were used. Hence, knowing the outer diameter of the damper $(50 \mathrm{~mm}$ ), we chose a commercial hydraulic cylinder with a wall thickness of 5 $\mathrm{mm}$ and internal diameter of $40 \mathrm{~mm}$ (Figures 2(c)|2(d)). The axial length of the cylinder is $192 \mathrm{~mm}$. The selected cylinder has its own cylinder head which is arranged for a piston rod diameter of $20 \mathrm{~mm}$ (Figure 2(a) 2(b) equipped with its own system of seals composed of a static seal, a rod seal and a wiper. The minimum axial length of the piston head was also fixed and had to be at least $L=90 \mathrm{~mm}$. That is because the piston head should be able to host the counter-rod for the entire stroke $(50 \mathrm{~mm})$ and there should be room also for the seal seat between them. The design path for MR damper involves principally four points:

- i) Selection of the input data (Table 11), determination of the geometrical constraints and the architecture (Figure 1).

- ii) Choice of a suitable commercial MRF.

- iii) Determination of the geometrical dimensions of the hydraulic system.

- iv) Design of the magnetic system.

\subsection{Design of the hydraulic system}

The input data are defined by the specifications and the choice of the commercial MR fluid. The design of the hydraulic system passes through the definition of the forces developed by the damper. The total force is the sum of three different 
contributions: the controllable force $F_{\tau}$ (Eq. 1), the viscous force $F_{\eta}$ (Eq. 2) and the dry friction forces, $F_{f}$, independent from velocity and mainly caused by the sealing system. According to (Yang 2001), the controllable force due to the MR fluid is equal to:

$$
F_{\tau}=c \frac{\tau_{y} L_{P t o t} A_{A}}{h}=\left(2.07+\frac{12 Q \eta}{12 Q \eta+0.4 w h^{2} \tau_{y}}\right) \frac{\tau_{y} L_{P t o t} A_{A}}{h} \operatorname{sign}\left(V_{D}\right)
$$

where $\tau_{y}$ is the yield stress of the fluid, $L_{P t o t}$ is the total axial activation length of the piston head, $A_{A}$ is the annular piston's area, $h$ is the fluid gap, $V_{D}$ id the piston velocity and $c$ is a coefficient depending on the volumetric flow rate $Q$, the viscosity of the fluid $\eta$ and the yield stress $\tau_{y}$. The viscous force is expressed by (Guglielmino et al.|2008):

$$
F_{\eta}=k \frac{12 \eta Q L A_{A}}{w h^{3}}=\left(1+\frac{w h V_{D}}{2 Q}\right) \frac{12 \eta Q L A_{A}}{w h^{3}}
$$

in which $L$ is the total axial length of the piston head, $w$ is the mean circumference of the damper's annular flow path and $k$ is a constant that depends on the volumetric flow rate and the velocity. The dynamic range $D_{R}$ is another important parameter to be considered during the design of magnetorheological damper as well as the analytical equations of the forces involved. $D_{R}$ is defined as the ratio between the total damper output force $F_{t o t}$ and the uncontrollable forces $F_{u n}$ (Eq. 3) (Yang 2001).

$$
D_{R}=\frac{F_{t o t}}{F_{u n}}=\frac{F_{\tau}+F_{\eta}+F_{f}}{F_{\eta}+F_{f}}=1+\frac{F_{\tau}}{F_{\eta}+F_{f}}
$$

The dynamic range provides an estimate of the influence of the control variable on the system response. So, an optimal design flow provides the maximum dynamic range. Considering the geometrical constraints, is possible to find the value of fluid gap that maximizes $D_{R}$. To do so, starting from the Eq. 1 and Eq. 2 and considering that the piston's annular area is defined as follow:

$$
A_{A}=\pi\left[\left(R_{A}-h\right)^{2}-R_{R}^{2}\right]
$$

the analytical equations of $F_{\tau}$ and $F_{\eta}$ can be rewritten as follow:

$$
F_{\tau}=\left(\frac{\tau_{y} L \alpha \pi\left[\left(R_{A}-h\right)^{2}-R_{R}^{2}\right]}{h}\right)
$$

where the activation length has been considered as $L_{P t o t}=\alpha L . \alpha$ must be minor than 1 because the axial space for the coil housings needs to be taken into account. A reasonable value is $\alpha=0.5$. As it can be seen in the schematic of Figure 3(a), $R_{A}$ and $R_{R}$ are the internal radius of the cylinder and the radius of the rod respectively.

$$
F_{\eta}=\left(\frac{12 V_{D} \pi\left[\left(R_{A}-h\right)^{2}-R_{R}^{2}\right] \eta L \pi\left[\left(R_{A}-h\right)^{2}-R_{R}^{2}\right]}{\pi\left(2 R_{A}-h\right) h^{3}}\right)
$$

even the coefficients $c$ and $k$ were manipulated but for the sake of brevity they are not reported. By substituting Equations 5 and 6 in 3 the analytical trend of the dynamic range as a function of the fluid gap can be obtained (Figure 4 ). The MR fluid used is the MR-140CG produced by Lord (Corporation 2014) with a viscosity of $\eta=0.280 \pm 0.070 \mathrm{~Pa} \cdot \mathrm{s}$. After setting up a yield stress $\tau_{y}=20 \mathrm{kPa}$, a velocity $V_{D}=0.15 \mathrm{~m} / \mathrm{s}$ and considering all the geometrical parameters such as $R_{A}=0.02$ $\mathrm{m}, R_{R}=0.01 \mathrm{~m}, L_{P t o t}=0.045 \mathrm{~m}, L=0.09 \mathrm{~m}$ (Figure 3(a) , the optimal fluid gap turned up to be $h=1.3 \mathrm{~mm}$ which gives $D_{R}=5.64$. After the consideration about the optimal fluid gap, we chose $h=1 \mathrm{~mm}$. Now, the non-controllable viscous force can be calculated as follow:

$$
F_{\eta}=\left(1+\frac{0.122 \times 1 \times 10^{-3} \times 0.15}{2 \times 1.6 \times 10^{-4}}\right) \times \frac{12 \times 0.28 \times 1.2 \times 10^{-4} \times 0.09 \times 8.19 \times 10^{-4}}{0.122 \times 0.001^{3}}=267.3 \mathrm{~N}
$$

Since the total force provided by the damper should be $F=F_{\tau}+F_{\eta}+F_{f}=2000 \mathrm{~N}$ and considering $F_{f}=150 \mathrm{~N}$, the required controllable force $F_{\tau}$ will be:

$$
F_{\tau}=2000-\left(F_{\eta}+F_{f}\right)=2000-(267.3+150)=1582.7 N
$$




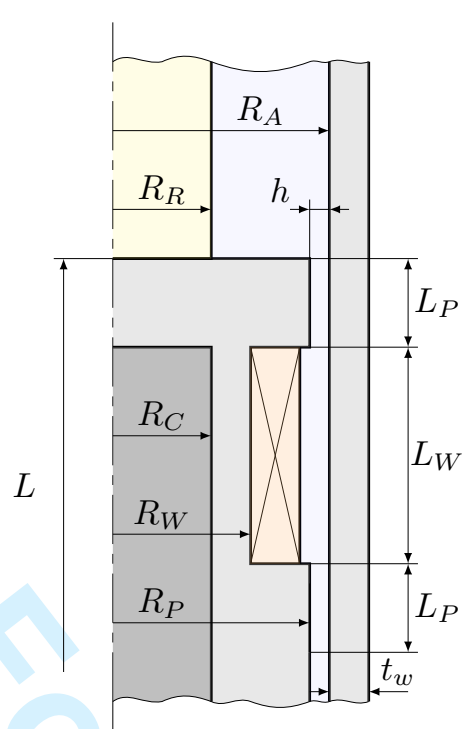

(a)

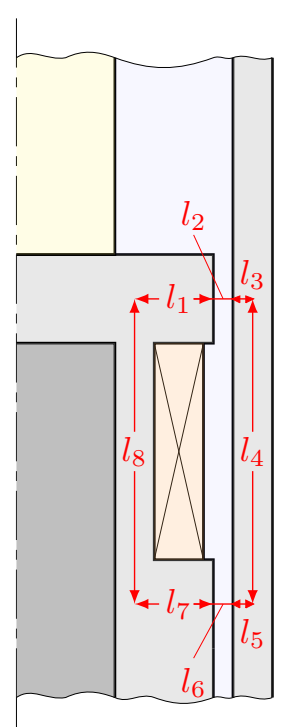

(b)

Figure 3. Half-section sketch of the hydraulic system. Main dimensions (a) and magnetic flux path (b).

Once the controllable force was found, with a yield shear stress $\tau_{y}=20 \mathrm{kPa}$, the total pole length is obtainable by means of Equation 9 .

$$
L_{P t o t}=\frac{F_{\tau} h}{c \tau_{y} A_{A}}=\frac{1582.7 \times 1}{2.36 \times 0.020 \times 819.54}=40.94 \mathrm{~mm}
$$

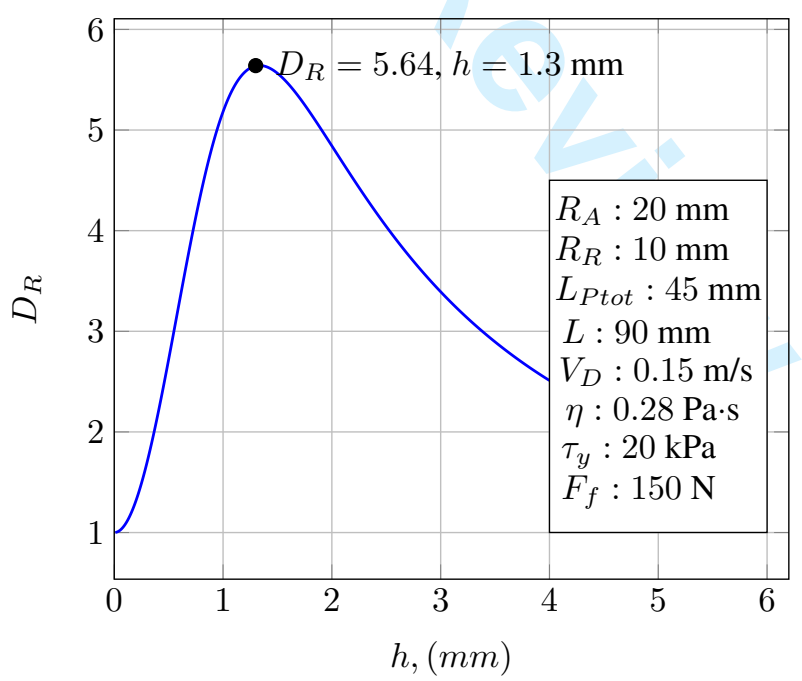

Figure 4. Dynamic range as a function of the fluid gap $h$.

Since there are two coil wires, the total number of activation areas are four (one at each ends of the piston head and two contiguous in the central part). Hence, the single activation's length is $L_{P t o t} / 4 \cong 10 \mathrm{~mm}$. The axial length of the coil housing is also obtainable:

$$
L_{W}=\left(L-L_{P t o t}\right) / 2=(90-40) / 2=25 \mathrm{~mm}
$$


Journal of Intelligent Material Systems and Structures 000(00)

Additional considerations can be drawn about the diameter of the inner piston chamber $R_{C}$. On the one hand, $R_{C}$ has to be big enough to host the counter-rod $\left(R_{R}=10 \mathrm{~mm}\right)$ and the two connecting leads of the coils. On the other hand, $R_{C}$ can not be too large because the material between the inner chamber and the coil housing would result to small, increasing the risk of saturation. We set $R_{C}$ to $11.5 \mathrm{~mm}$, according also with the magnetic simulations described in subsection 2.2.1.

\subsection{Design of the magnetic system}

The aim in designing the magnetic circuit is to determine the necessary amp-turns $(N I)$ that provides the required magnetic field intensity with the working current. An optimal design of the magnetic circuit requires to reach the desired magnetic field induction in the fluid gap while minimize the energy lost in the steel flux conduit and regions of non-working area. The design starts from the knowledge of the characteristics of the working fluid. As reported in the previous paragraph, the MR fluid considered in this work is the commercial MRF-140CG produced by Lord Corporation. The magnetic B-H relationship of a MR fluid can be defined as (Wereley|2013):

$$
B=1.91 \phi^{1.133}\left[1-e^{\left(-10.97 \mu_{0} H_{m r f}\right)}\right]+\mu_{0} H_{m r f}
$$

where, $B$ is in Tesla, $H_{m r f}$ is in $\mathrm{A} / \mathrm{m}$, and $\mu_{0}=0.000001256 \mathrm{H} / \mathrm{m} . \phi$ is the iron particle volume percentage in the MR fluid. The yield shear stress $\tau_{y}\left(H_{m r f}\right)$ is given by the experimentally derived equation from (Lee et al. 2013, Yoo and Wereley 2002) and depends on the magnetic field intensity and the particle volume fraction $\phi$ :

$$
\tau_{y}\left(H_{m r f}\right)=271700 \phi^{1.5239} \tanh \left(6.33 \times 10^{-6} H_{m r f}\right)
$$

$C$ is a coefficient dependent on the carrier fluid of the MR fluid $(C=1$ for hydrocarbons, 1.16 for water and 0.95 for silicone oils). Using Equations 11 and 12 is possible to obtain the values of magnetic induction $B$, relative magnetic permeability $\mu_{r}$ as well as the yield shear stress $\tau_{y}\left(H_{m r f}\right)$, as a function of the magnetic field intensity for the MRF-140CG. Considering the desired yield stress $\tau_{y}=20 \mathrm{kPa}$ and the relation described by Equation 12, the required magnetic field intensity $H_{m r f}$ is $50 \mathrm{kA} / \mathrm{m}$. Then, using Equation 11 the magnetic field induction along the fluid gap is $B_{m r f}=350 \mathrm{mT}$. Subsequently, since the magnetic induction flux remains constant through all the circuit length, by means of the Kirchoff's law of magnetic circuits, the required value of amp-turns $(N I)$ is obtainable as follow (Yang|2001, Gavin et al.|2001):

$$
N I=\sum H_{i} l_{i}=H_{m r f} h+\sum H_{\text {steel }} l_{i}
$$

where $h$ is the fluid gap and $l_{i}$ the single length of each links which compose the circuit $3(\mathrm{~b})$. In order to reach the best performances, the material which composes the magnetic circuit should have high magnetic permeability and high magnetic saturation. A material with such properties is the AISI 1010, which is a low-carbon steel $(\mathrm{C} \%<0.10)$. This material though, is hardly available because of is being used for niche applications. Hence the AISI 430 was used. AISI 430 is a ferritic stainless steel with a high relative magnetic permeability of about 800 . Considering a nominal working current of $1 \mathrm{~A}$ the required total number of coil wires is $N_{t o t}=320$. Which means a number of wires for each coil of $N=160$. In this work we considered a copper wire with a diameter of $d_{w}=0.75 \mathrm{~mm}$. We decided to set the inner coil radius $R_{\text {Icoil }}=15 \mathrm{~mm}$ hence the outer coil radius turned out $R_{\text {Ocoil }}=18.4 \mathrm{~mm}$. The resistance of the single coil wire can also be calculated knowing the wire length and its sectional area and it is $R_{\text {coil }}=0.7 \Omega$.

2.2.1. Magnetic Simulation Before the assembly of the prototype, a magnetic finite element analysis was performed. This task was responsible of two contributes. First, comparing the simulated values of magnetic field with the calculated ones. Second, verifying that the magnetic field was below the saturation level even in the thinnest section of the circuit between the radii of the inner coil and the inner chamber of the piston head. The software FEMMv4.2 (Meeker 2015) was adopted to perform all the simulations. Figure 5(a) shows the discretized axial-symmetric model of the magnetic circuit 
which comprehends part of the piston head, the flange and the cylinder's wall. The materials were set using the FEMM material library. The AISI 430 was used for the piston head and the flange. The AISI 1020 was used for the cylinder. For the MR fluids, a new material was set up with the magnetic properties described by Equation 11. Figures 5(b) and 5(c) represent the path of the magnetic flux and the values of the magnetic field density resulted with a working current of $1 \mathrm{~A}$. As it can be seen the values of $B$ computed by the simulation are lower than $1.2 \mathrm{~T}$ that is a critical point after which begins saturation. From the design of the hydraulic system, a yield stress $\tau_{y}=20 \mathrm{kPa}$ was set, considering a current of $1 \mathrm{~A}$. That implied a magnetic field of $B_{m r f}=350 \mathrm{mT}$ along the activation areas. Figure 5(e) depicts the values of $B$ through the dash-dotted red line along one of the activation gaps (Figure 5(d) . The simulated values are slightly lower than those obtained from the analytical calculation. A reasonable explanation is that the number of coil wires considered is a little less than those calculated, since the coiling was handmade in our Lab.

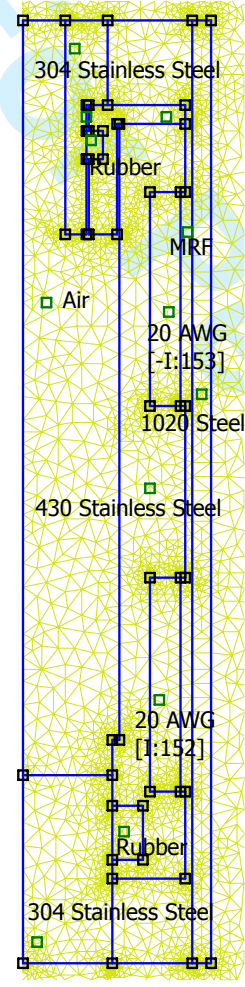

(a)

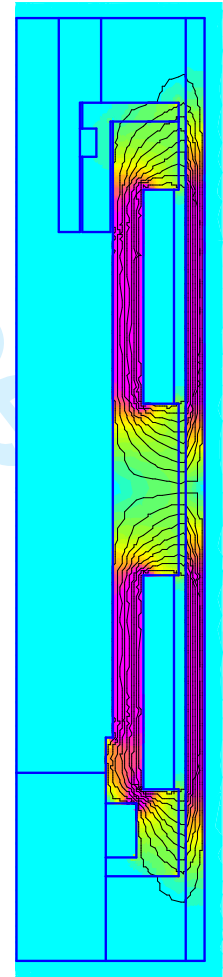

(b)

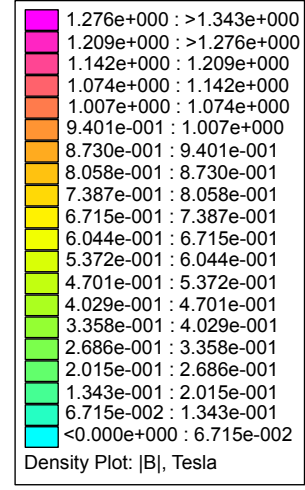

(c)

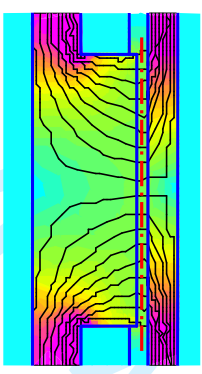

(d)

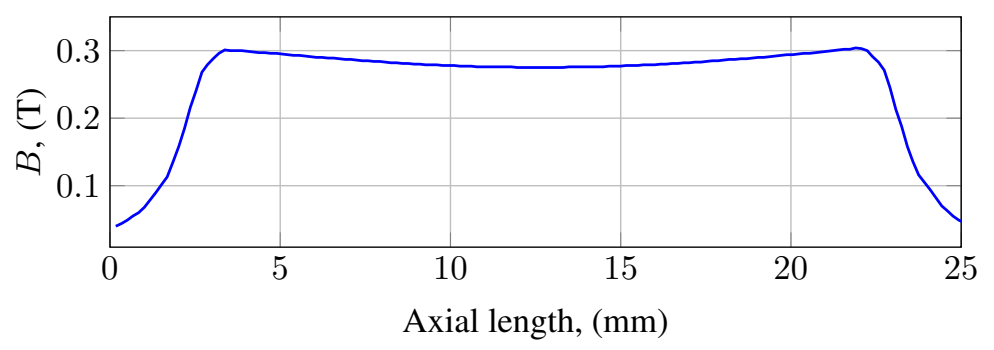

(e)

Figure 5. 2D FEMM model of the piston head (a), magnetic field values (b) and relative legend (c). Magnified image of the activation gap (d). Graph of the magnetic field (e) across the red line in (d). 


\subsection{Prototype Manufacturing}

2.3.1. Main system Figure 6 reports the section view of the MR damper with all its components. After the design of the hydraulic and magnetic systems, the piston head $(E)$, the counter-rod $(H)$ along with the end plug $(M)$ and the upper rod $(A)$ were manufactured. The upper rod and the counter-rod were both made of brass because they had to be non-magnetic in order not to influence the magnetic field during piston moving. The final piston head is composed of two parts: the main body $(E)$ and a flange $(C)$. This is to facilitate the whole process of assembly. The main body hosts the two coil housing $(I)$, the inner chamber $(F)$ and the seal seat. The dynamic rod seal $(L)$ allows the counter-rod to slide inward the inner chamber of the piston head avoiding the passage of the fluid through it. The flange has two main aims: linking the main body of the piston head to the upper rod and permits the coil wire to pass through it. Even the flange is equipped with two static nitrile-rubber seal (O-ring). The linkage is made by four M4 cap screw and a M12 drilled screw $(D)$ for letting the coil wire coming from the narrow passage $(G)$ to pass through it. The end plug was bonded to the cylinder $(B)$ using an anaerobic adhesive (LOCTITE 638). Finally, the MRF was poured manually through the welded boss.

2.3.2. Pressurization system The aim of the pressurization system is the active regulation of the fluid pressure. The presented system is capable of doing this in a totally controllable manner without the aid of volumetric pumps, which are incompatible with MR fluids because of the too high viscosity. The new manufactured system is composed of a screw-drive 
system in which an adjustment pin $(R)$ controls the axial movement of a cursor $(O)$. As it can be seen schematically in Figure 6, as the adjustment pin is rotating into its housing $(Q)$, a square profile $(P)$ does not allow the cursor to rotate forcing it to slide axially. Therefore, the cursor constricts the fluid in a smaller volume causing the pressure to increase. Moreover, the present system is able to maintain the position of the cursor as well as the pressure level without a continuous energy supply. Indeed, the threaded parts were designed to prevent retrograde motion. Finally, a pressure transducer can be install in $(N)$ to monitor the internal pressure.

\section{EXPERIMENTAL TEST}

\subsection{Experimental plan}

The design of experiment methodology was adopted to arrange the experimental test. The damper was tested under sinusoidal displacements. The variables involved are amplitude $A$ and frequency $f$ of the sinusoidal input, current $I$ and pressure level $p$. Three amplitude levels were chosen, $5 \mathrm{~mm}, 10 \mathrm{~mm}$ and $15 \mathrm{~mm}$. The values of frequency were $1 \mathrm{~Hz}$ and 2 Hz. In this way, the maximum velocity value $\left(V_{\max }=2 \pi A f\right)$ is ranging between $31.41 \mathrm{~mm} / \mathrm{s}$ (with $A=5 \mathrm{~mm}$ and $f=1$ $\mathrm{Hz}$ ) and $188.49 \mathrm{~mm} / \mathrm{s}$ (with $A=15 \mathrm{~mm}$ and $f=2 \mathrm{~Hz}$ ). Three levels for current $I, 0,1$ and $2 \mathrm{~A}$. Three values even for the pressure level which are 0,20 and 40 bar. For each combination of variables and levels, three replicates were carried out with a consequence of 162 total experimental tests. A summary of the variables used and their values are reported in Table 2

Table 2. Experimental plan.

\begin{tabular}{lccc}
\hline Levels & I & II & III \\
\hline Amplitude, $A, \mathrm{~mm}$ & 5 & 10 & 15 \\
\hline Frequency, $f, \mathrm{~Hz}$ & 1 & 2 & \\
\hline Current, $I, \mathrm{~A}$ & 0 & 1 & 2 \\
\hline Pressure, $p$, bar & 0 & 20 & 40 \\
\hline Replicates & 3 for each combination \\
\hline Experimental Points & \multicolumn{3}{c}{54} \\
\hline Grand total & \multicolumn{3}{c}{162} \\
\hline
\end{tabular}

\subsection{Experimental set up}

The test were carried out using the universal testing machine MTS Mini Bionix. The damper was placed between the servo hydraulic grips of the machine using two ball joints (screwed to the upper rod and the end-plug) and calibrated pins. The sinusoidal inputs and their parameters were set using the related control software of the machine. Each test lasted for 20 cycles with a sampling rate of $512 \mathrm{~Hz}$. The output data were the vectors of time $t$, measured force $\mathrm{F}$ and the sinusoidal displacement $x$. The current was supplied in DC mode using a stabilized TTi power supply system. The internal pressure level was set manually using the adjustment pin and measured by means of a piezoresistive pressure transducer by Keller (Series $25 \mathrm{Y}$, (Keller 2015), Figure 7). The output signal of the transducer is based on an analogue $4-20 \mathrm{~mA}$ current loop in which $4 \mathrm{~mA}$ represents the lowest end of the range and $20 \mathrm{~mA}$ the highest. The main advantage of the current loop is that the accuracy of the signal is not affected by voltage drop in the interconnecting wiring. Anyway, the appropriate conditioning was needed in order to obtain the effective pressure values. This operation was done using a simple circuit that converts the current range between 4 and $20 \mathrm{~mA}$ into a voltage range between 0 and 5 Volts. After that, the voltage output was translated into a digital value using a 10 bit A/D converter (ArduinoUNO 2015). During the test, the actual values of pressure were visualized on monitor and then saved in a text file. 


\section{RESULTS AND DISCUSSIONS}

Figures 8(a), 8(b) 8(c) show the experimental force as a function of the applied sinusoidal displacements. At a glance, it can be seen that especially at low speeds ( $A=5 \mathrm{~mm}$ and $f=1 \mathrm{~Hz}$ ) cavitation can be observed. This phenomenon manifests as a delay in force caused by the presence of air bubbles. These air bubbles occur when there is too little magnetorheological fluid in the damper or are produced when the pressure drop through the piston valve is higher than the pressure in the damper. Air dispersed in the fluid generate a lag in the force response during the changes of direction of the damper (graphically represented by the two steps in the force trend during the zero-crossing). When the bubbles collapse MRFs return incompressible again and the force response returns to its normal value. The increases of the pressure level causes the gas bubbles to collapse and dissolve in the fluid, making the cavitation almost disappear. Increasing the velocity up to $188.49 \mathrm{~mm} / \mathrm{s}$ ( $A=15 \mathrm{~mm}$ and $f=2 \mathrm{~Hz}$ ) the force response becomes less sensitive to this phenomenon. In fact, the higher velocity caused a greater dispersion of the air bubbles and consequently a lower force lag. The influence of an hydrostatic pressure on the behaviour of MRFs resulted less strong compared to a unidirectional compression (Guo et al. 2013, Becnel and Wereley 2013). Indeed, in our case the isotropic state of compression does not allow the ferromagnetic particles to organize themselves into thicker columns and increase the yield stress of MRFs. It might be assumed that the change in the force response considering different values of internal pressure is due to the higher friction exerted by the seals system. The experimental values of the force response for these preliminary tests are reported in Table 3

\subsection{Equivalent viscous damping model}

In order to evaluate the phenomenon of cavitation the results were compared with a simple damping model. In particular, the equivalent viscous damping model was taken into account (Sapiski 2005). The equivalent damping coefficient is calculated through the dissipated energy of the damper, $E$, in one cycle, which represents the area enclosed in the force-displacement 


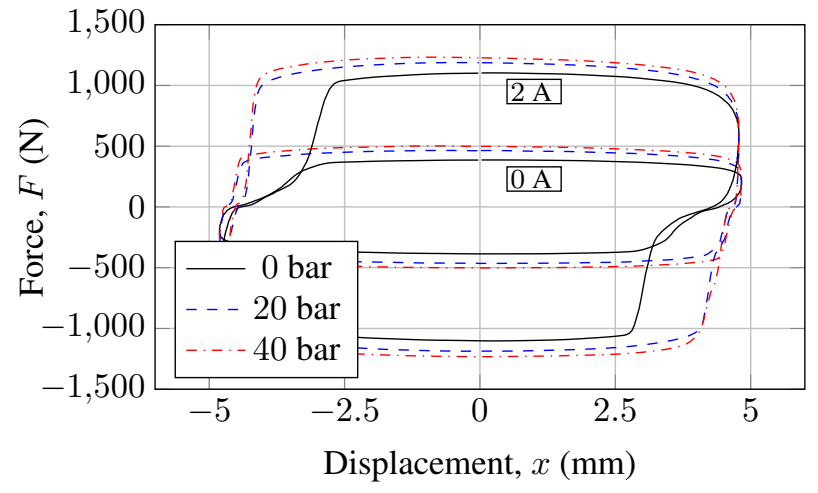

(a)

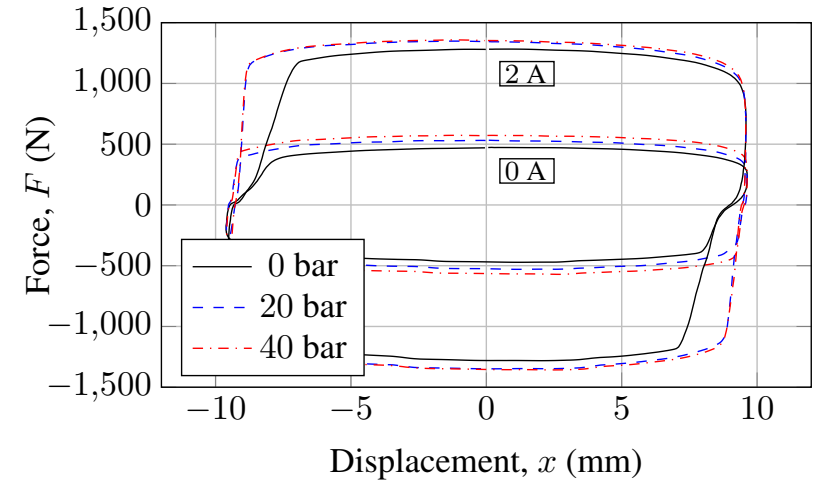

(b)

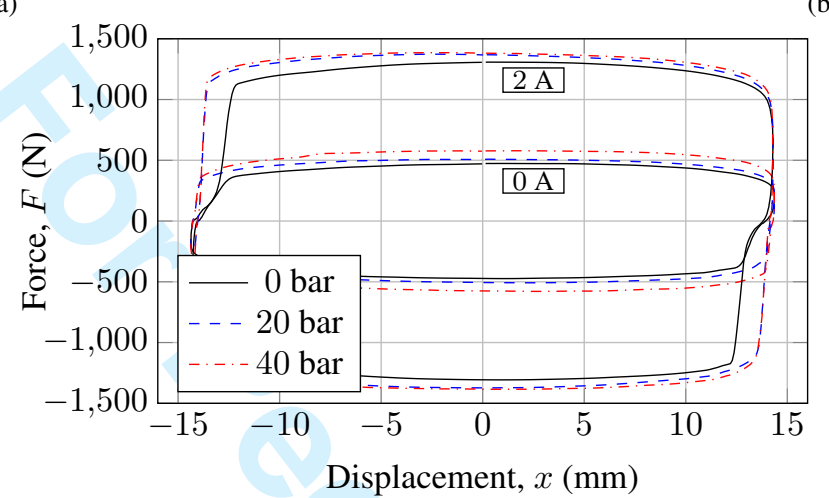

(c)

Figure 8. Experimental force-displacement curves for current $0 \mathrm{~A}$ and $2 \mathrm{~A}$. Pressure values 0 (solid lines), 20 (dotted lines) or $40 \mathrm{bar}$ (dash-dotted lines). Amplitude of 5, 10 and $15 \mathrm{~mm}$ reported in (a), (b) and (c) respectively.

Table 3. Experimental maximum values of force response, $F(\mathrm{~N})$.

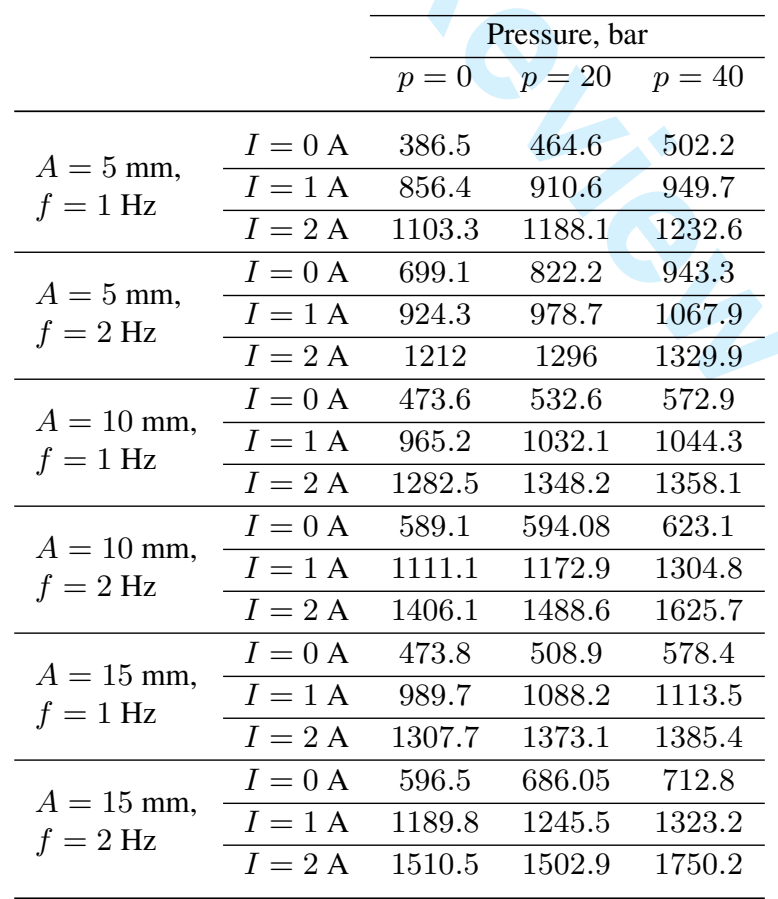


2

3

4

graph. Therefore, for the calculation of the dissipated energy we considered the difference between the maximum, $F_{\text {max }}$, and the minimum force, $F_{m i n}$, times the amplitude, $A$, for each level of current and pressure, according to:

$$
E=2\left(F_{\max }-F_{\min }\right) A
$$

As for the equivalent viscous damping model, we calculated the dissipated energy related to the experimental tests by means of an algorithm on Matlab that calculates the value of the area enclosed in a given plot. We compared the experimental dissipated energies and the ones related to the viscous model in Figure 9. Thus, we assessed how the internal pressure and the current affect cavitation. In Table 4 we reported the relative errors between the energies obtained from the simplified equivalent damping model and the experimental tests considering the total damping loop force and calculated as $e \%=\frac{E_{\text {model }}-E_{e x p}}{E_{\text {model }}} \times 100$. Moreover, considering the average between the error values at the same pressure level and regrouping them for each current level (Figure 10, it can be seen that the higher is the pressure, the better the equivalent damping model approximates the experimental damping energy, confirming the considerations brought up in the Paragraph 4

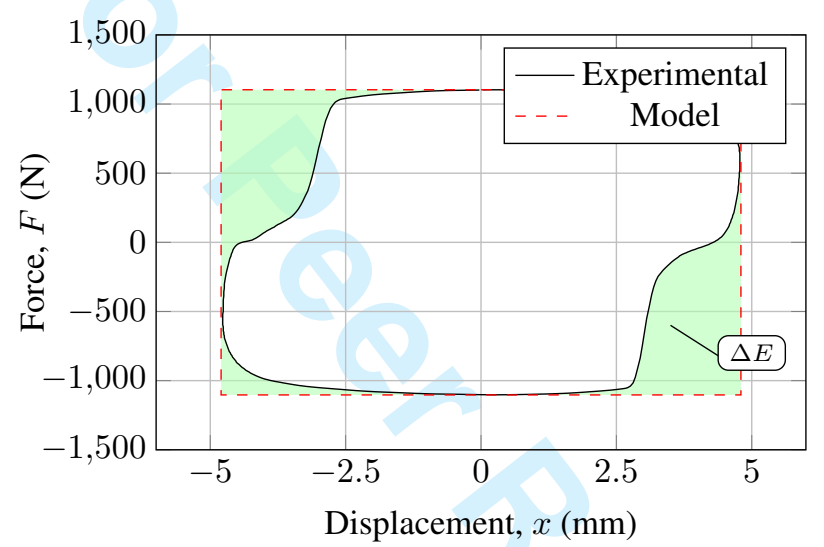

Figure 9. Comparision between experimental curves and equivalent damping model with no pressure applied, at 2 A, frequency of $1 \mathrm{~Hz}$ and amplitude of $5 \mathrm{~mm}$.

\begin{tabular}{lcccc}
\cline { 3 - 5 } & & \multicolumn{3}{c}{ Pressure, bar } \\
\cline { 2 - 5 } & & $p=0$ & $p=20$ & $p=40$ \\
\hline \multirow{2}{*}{$\begin{array}{l}A=5 \mathrm{~mm}, \\
f=1 \mathrm{~Hz}\end{array}$} & $I=0 \mathrm{~A}$ & 17 & 9 & 8 \\
\cline { 2 - 5 } & $I=1 \mathrm{~A}$ & 18 & 10 & 9 \\
\cline { 2 - 5 }$I=2 \mathrm{~A}$ & 21 & 10 & 10 \\
\hline \multirow{2}{*}{$\begin{array}{l}A=5 \mathrm{~mm}, \\
f=2 \mathrm{~Hz}\end{array}$} & $I=0 \mathrm{~A}$ & 21 & 11 & 13 \\
\cline { 2 - 5 } & $I=1 \mathrm{~A}$ & 21 & 11 & 10 \\
\hline \multirow{2}{*}{$\begin{array}{l}A=10 \mathrm{~mm}, \\
f=1 \mathrm{~Hz}\end{array}$} & $I=0 \mathrm{~A}$ & 21 & 11 & 11 \\
\cline { 2 - 5 } & $I=1 \mathrm{~A}$ & 12 & 8 & 7 \\
\hline \multirow{2}{*}{$\begin{array}{l}A=10 \mathrm{~mm}, \\
f=2 \mathrm{~Hz}\end{array}$} & $I=0 \mathrm{~A}$ & 13 & 7 & 7 \\
\cline { 2 - 5 } & $I=1 \mathrm{~A}$ & 13 & 9 & 9 \\
\hline \multirow{2}{*}{$\begin{array}{l}A=15 \mathrm{~mm}, \\
f=1 \mathrm{~Hz}\end{array}$} & $I=0 \mathrm{~A}$ & 13 & 8 & 8 \\
\cline { 2 - 5 } & $I=1 \mathrm{~A}$ & 10 & 7 & 6 \\
\hline \multirow{2}{*}{$\begin{array}{l}A=15 \mathrm{~mm}, \\
f=2 \mathrm{~Hz}\end{array}$} & $I=2 \mathrm{~A}$ & 10 & 6 & 6 \\
\cline { 2 - 5 } & $I=1 \mathrm{~A}$ & 13 & 11 & 10 \\
\cline { 2 - 5 } & $I=2 \mathrm{~A}$ & 11 & 8 & 8
\end{tabular}

Table 4. Relative \% error of the experimental values and friction model. 


\subsection{Analysis of variance}

The design of experiment adopted is a powerful statistical technique based on the analysis of variance (ANOVA, Anderson and Whitcomb 2007)), designed appositely for the analysis of experimental tests. ANOVA calculates the variance of the observed variable taking into account a specific input variable and the global variance of the response. Figure 11 shows the half-normal probability plot provided by Design Expert 8.0 (Stat-Ease 2015). The X-axis represents the standardized effect of each factor involved (yellow squares) (Table 2). The greater is the value associated with a variable, the higher is its influence on the response. The values on the Y-axis are not based on the DOE data. They are given by the idealized expected values for this number of effects if they were drawn from a half-normal distribution. The factors on the red line are statistically normal (follow a stochastic law) so they do not affect the response, like the sum of errors (green triangles).

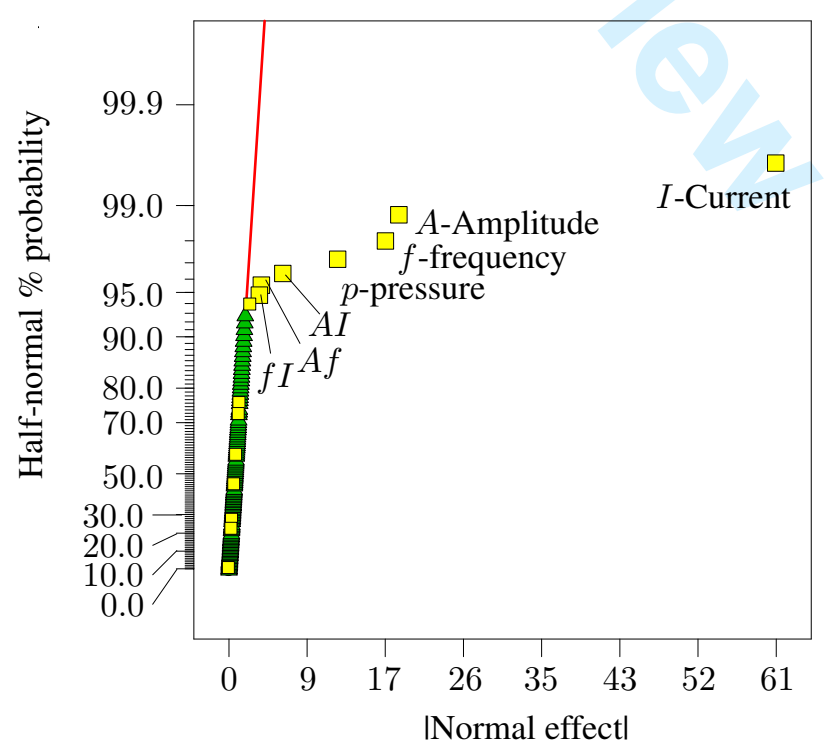

Figure 11. Half-normal probability plot representing the effect of the variables on the force response of the damper. 
The maximum force is influenced by the frequency, the amplitude, the current and the internal pressure, as expected and the half-normal probability plot helps in understanding which are the most important factors. The force response is mostly influenced by the current value $(I)$ as reported in Figure 11 Less important are the effects of the sinusoidal input's parameters (amplitude $A$ and frequency $f$ ), the internal pressure $(p)$ and their interactions $(A I, A f, f I)$. The preliminary tests on the presented magnetorheological damper brought up several aspects, compared to the existing architectures. In single-rod dampers the upper surface and the lower surface of the piston head are different so that the force exerted is different as well in compression and rebound. The new architecture instead, like the through-rod damper, has the same annular area so it behaves in the same manner both in compression and rebound. This aspect shows as a main benefit the lack of the offset between positive and negative forces (centered force-displacement graph). In single-rod dampers the accumulator pressure acts on the rod area and produces a static force independent from velocity. On the contrary, in this new architecture there is no gas force pushing the piston rod out of the damper body. The through-rod architecture (Yang 2001), avoids the displacement volume issue by having a passing through-rod which causes no volume variation. However, it presents some disadvantages. First, there are external seals at both ends subject to high pressures that may cause lack of fluid. Second, the protruding free end may be inconvenient or dangerous, especially in application like steering dampers. The design architecture with its internal counter-rod avoids this problem. Third, the problem of the thermal expansion of the fluid can be overcome thanks to the designed pressurization system that acts directly on the fluid volume. Moreover, contrarily to traditional mono-tube and twin-tube dampers, the architecture proposed allows no restrictions in installation orientation.

\section{CONCLUSION}

This paper investigates the behaviour of magnetorheological fluids when subjected to a hydrostatic pressure through the study of a new magnetorheological damper which is able to control its internal pressure. We designed a novel architecture that differs from the existing ones by the presence of an internal counter-rod placed at the bottom of the damper. The additional counter-rod, sliding into the piston head eliminates every flexible elements used to compensate the volume changing and then permits the pressure to be controlled. A pressurization system provides an internal pressure control up to 40 bar. An experimental campaign was performed to assess the behaviour of the new damper under a sinusoidal inputs and several values of magnetic field and internal pressure. The influence of the hydrostatic pressure on the yield stress of magnetorheological fluids was estimated by the measured force response. The preliminary test proved that the new prototype is able to work without the volume compensator and also enables a precise internal pressure control. Even though the benefit of pressure is evident in terms of preventing cavitation, the influence of the hydrostatic pressure on the yield stress of the MR fluid is less appreciable. To the best of our knowledge, the magnetorheological damper we designed and manufactured presents a novel architecture with several advantages, such as no protruding elements, thermal compensation system and cavitation prevention and may suggest further studies that could lead to several potential improvements for the MR technology.

\section{References}

Anderson, M. J. and Whitcomb, P. J. (2007), DOE Simplified: Practical Tools for Effective Experimentation, Productivity Pr. ArduinoUNO (2015), 'http://www.arduino.cc/en/Main/arduinoBoardUno. (accessed 20 Feb 2015)'.

Becnel, A. C., Sherman, S. G., Hu, W. and Wereley, N. M. (2015), 'Squeeze strengthening of magnetorheological fluids using mixed mode operation', Journal of Applied Physics 117(17), 17C708.

Becnel, A. C. and Wereley, N. M. (2013), Demonstration of combined shear and squeeze strengthening modes in a searle-type magnetorheometer, in 'Volume 1: Development and Characterization of Multifunctional Materials; Modeling, Simulation and Control of Adaptive Systems; Integrated System Design and Implementation', ASME, p. V001T03A036. 
Carlson, J. D. and Jolly, M. R. (2000), ‘Mr fluid, foam and elastomer devices', Mechatronics 10(4-5), 555-569.

Corporation, L. (2014), 'Mrf-140cg magnetorheological fluid. technical datasheet: http://www.lordfulfillment.com/upload/ds7012.pdf. (accessed 20 feb 2015)'.

Gavin, H., Hoagg, J. and Dobossy, M. (2001), Optimal design of mrf dampers, in 'U.S. - Japan Workshop on Smart Structures for Improved Seismic Performance in Urban Regions', number August, Seattle WA, pp. 225-236.

Guglielmino, E., Sireteanu, T., Stammers, W. C., Ghita, G. and Giuclea, M. (2008), Semi-active Suspension Control, Springer London, London.

Guo, C., Gong, X., Xuan, S., Qin, L. and Yan, Q. (2013), 'Compression behaviors of magnetorheological fluids under nonuniform magnetic field', Rheologica Acta 52(2), 165-176.

Jolly, M. R., Bender, J. W. and Carlson, J. D. (1999), 'Properties and applications of commercial magnetorheological fluids', Journal of Intelligent Material Systems and Structures 10(1), 5-13.

Keller (2015), 'Piezoresistive pressure transucer series 25y -http://www.keller-druck.com/home_e/paprod_e/23y_25y_e.asp. (accessed 20 feb 2015)'.

Lau, Y. K. and Liao, W. H. (2007), 'Pressurized magnetorheological damper - us20070023245 a1'.

Lee, J.-H., Han, C., Ahn, D., Lee, J. K., Park, S.-H. and Park, S. (2013), 'Design and performance evaluation of a rotary magnetorheological damper for unmanned vehicle suspension systems.', TheScientificWorldJournal 2013, 894016.

Mazlan, S., Ekreem, N. and Olabi, A. (2008), 'An investigation of the behaviour of magnetorheological fluids in compression mode', Journal of Materials Processing Technology 201(1-3), 780-785.

Meeker, D. (2015), 'Femm 4.2 - finite element method magnetics homepage.', http://www.femm.info/wiki/HomePage .

Sapiski, B. (2005), 'Linearized characterization of a magnetorheological fluid damper.', Mechanics / AGH University of Science and Technology. 24(no. 2), 144-149.

Spaggiari, A. and Dragoni, E. (2012), 'Effect of pressure on the flow properties of magnetorheological fluids', Journal of Fluids Engineering, Transaction of the ASME 134(9)(262).

Spaggiari, A. and Dragoni, E. (2013), 'Combined squeeze-shear properties of magnetorheological fluids: Effect of pressure', Journal of Intelligent Material Systems and Structures 25(9), 1041-1053.

Stat-Ease (2015), 'Design expert 8.0. available at: www.statease.com. (accessed 19 jan 2015)'.

Tang, X., Zhang, X., Tao, R. and Rong, Y. (2000), 'Structure-enhanced yield stress of magnetorheological fluids', Journal of Applied Physics 87(5), 2634.

Tian, Y., Meng, Y., Mao, H. and Wen, S. (2002a), 'Electrorheological fluid under elongation, compression, and shearing.', Physical review E: Statistical, nonlinear, and soft matter physics 65(3 Pt 1), 031507.

Tian, Y., Meng, Y., Mao, H. and Wen, S. (2002b), 'Mechanical property of electrorheological fluid under step compression', Journal of Applied Physics 92(11), 6875.

Tian, Y., Zhang, M., Zhu, X., Meng, Y. and Wen, S. (2010), 'Ultrahigh yield stress in a general electrorheological fluid under compression', Smart Materials and Structures 19(3), 035009.

Wereley, N. M. (2013), Magnetorheology: Advances and Applications, Royal Society of Chemistry.

Yang, G. (2001), Large-scale magnetorheological fluid damper for vibration mitigation: modeling, testing and control, PhD thesis, University of Notre Dame.

Yoo, J.-H. and Wereley, N. M. (2002), 'Design of a high-efficiency magnetorheological valve', Journal of Intelligent Materials Systems and Structures 13(10), 679-685.

Zhang, X. Z., Gong, X. L., Zhang, P. Q. and Wang, Q. M. (2004), 'Study on the mechanism of the squeeze-strengthen effect in magnetorheological fluids', Journal of Applied Physics 96(4), 2359.

Zhu, X., Jing, X. and Cheng, L. (2012), 'Magnetorheological fluid dampers: A review on structure design and analysis', Journal of Intelligent Material Systems and Structures 23(8), 839-873. 


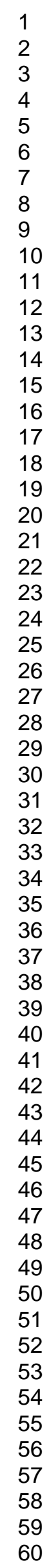

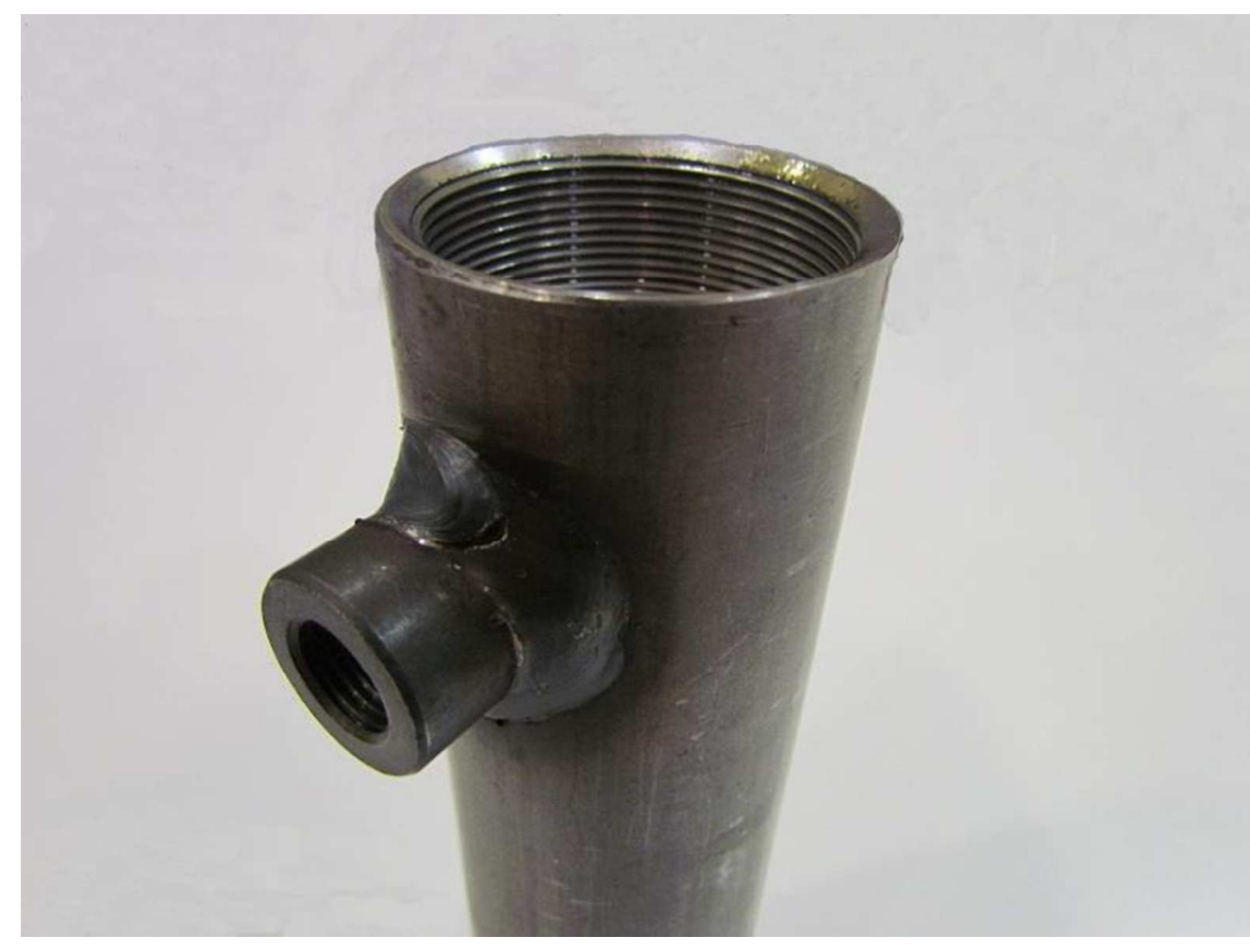

$112 \times 84 \mathrm{~mm}(180 \times 180 \mathrm{DPI})$

http://mc.manuscriptcentral.com/jimss 


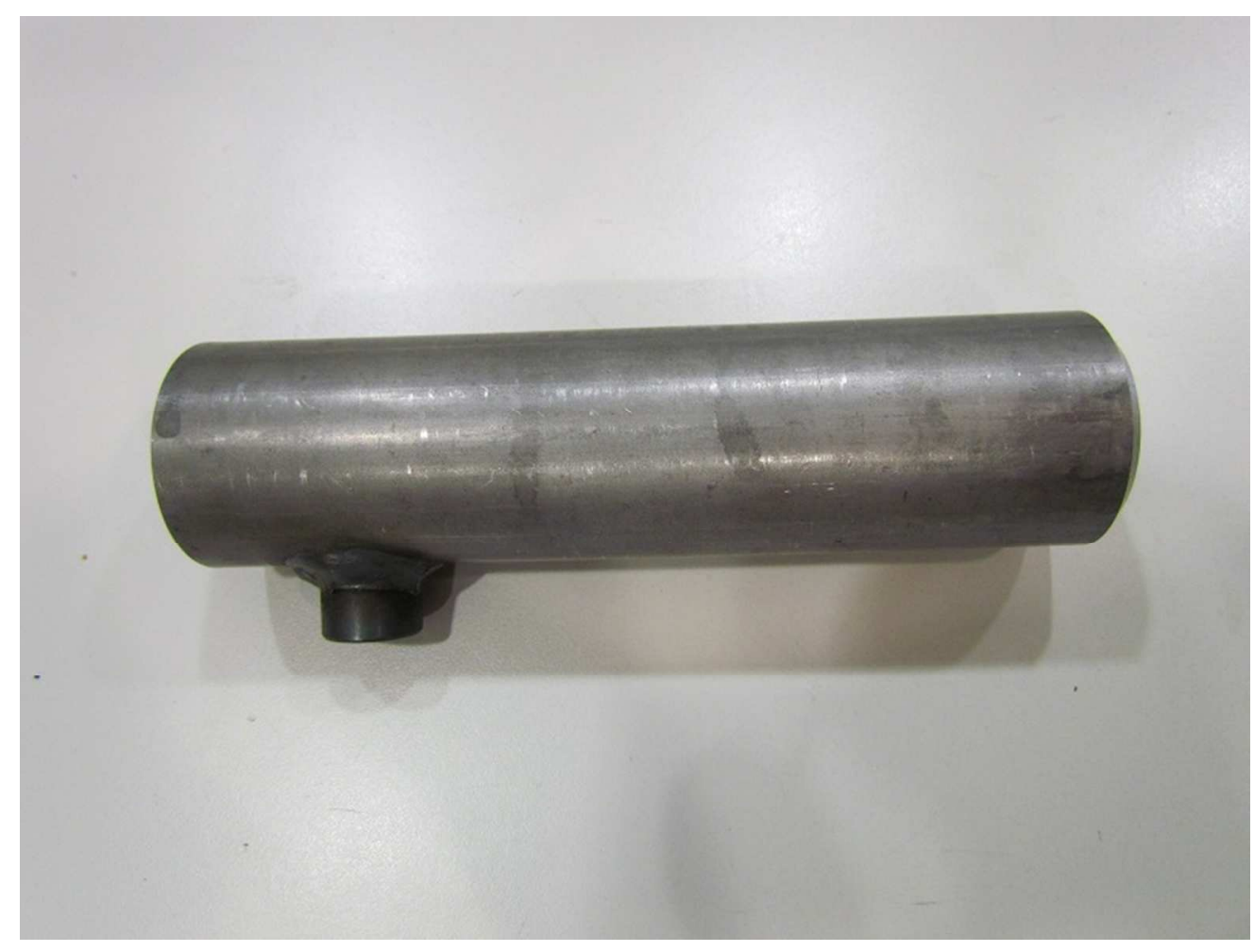

$112 \times 84 \mathrm{~mm}(180 \times 180 \mathrm{DPI})$ 


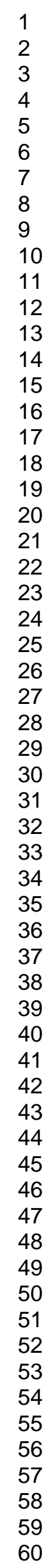

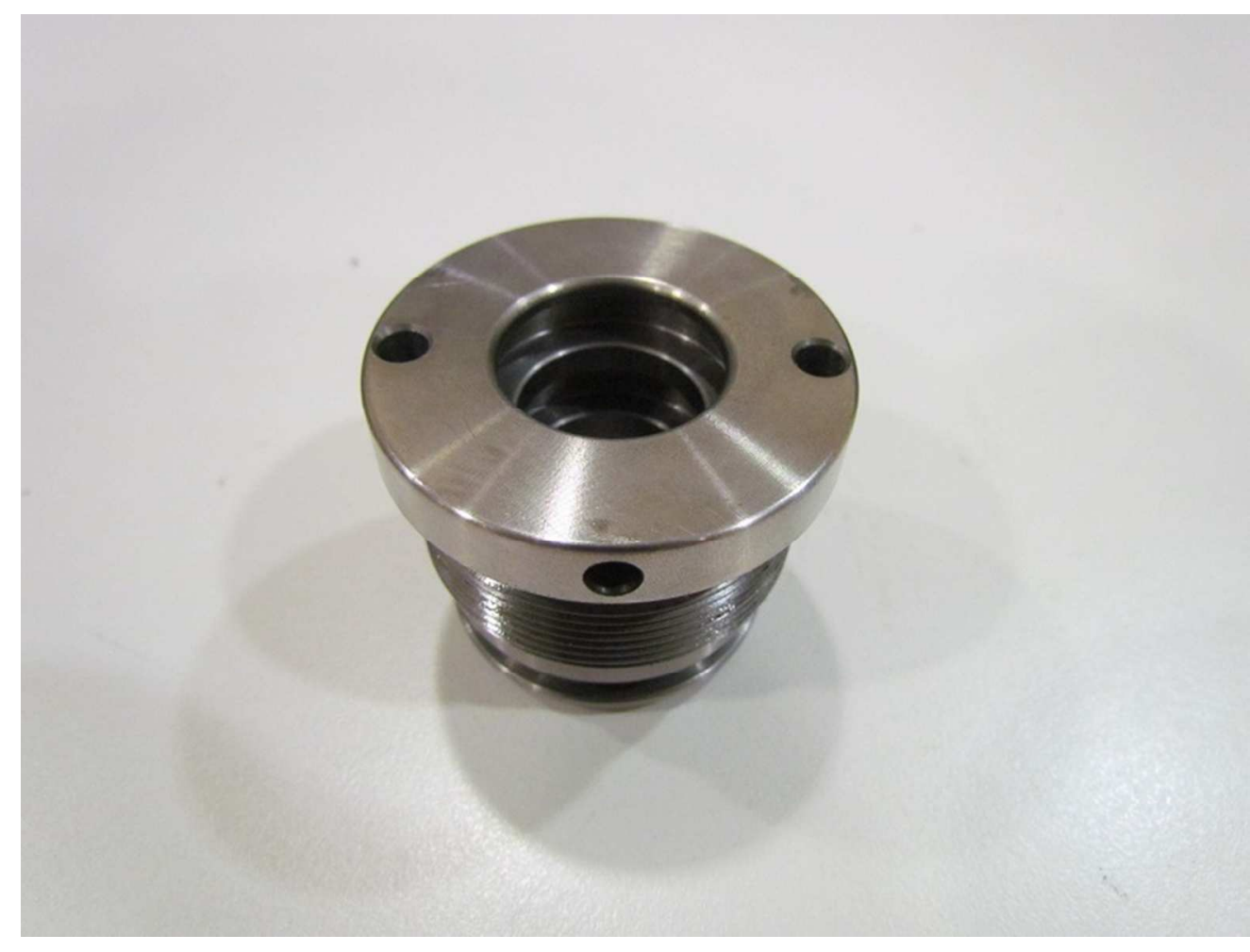

$112 \times 84 \mathrm{~mm}(180 \times 180 \mathrm{DPI})$

http://mc.manuscriptcentral.com/jimss 


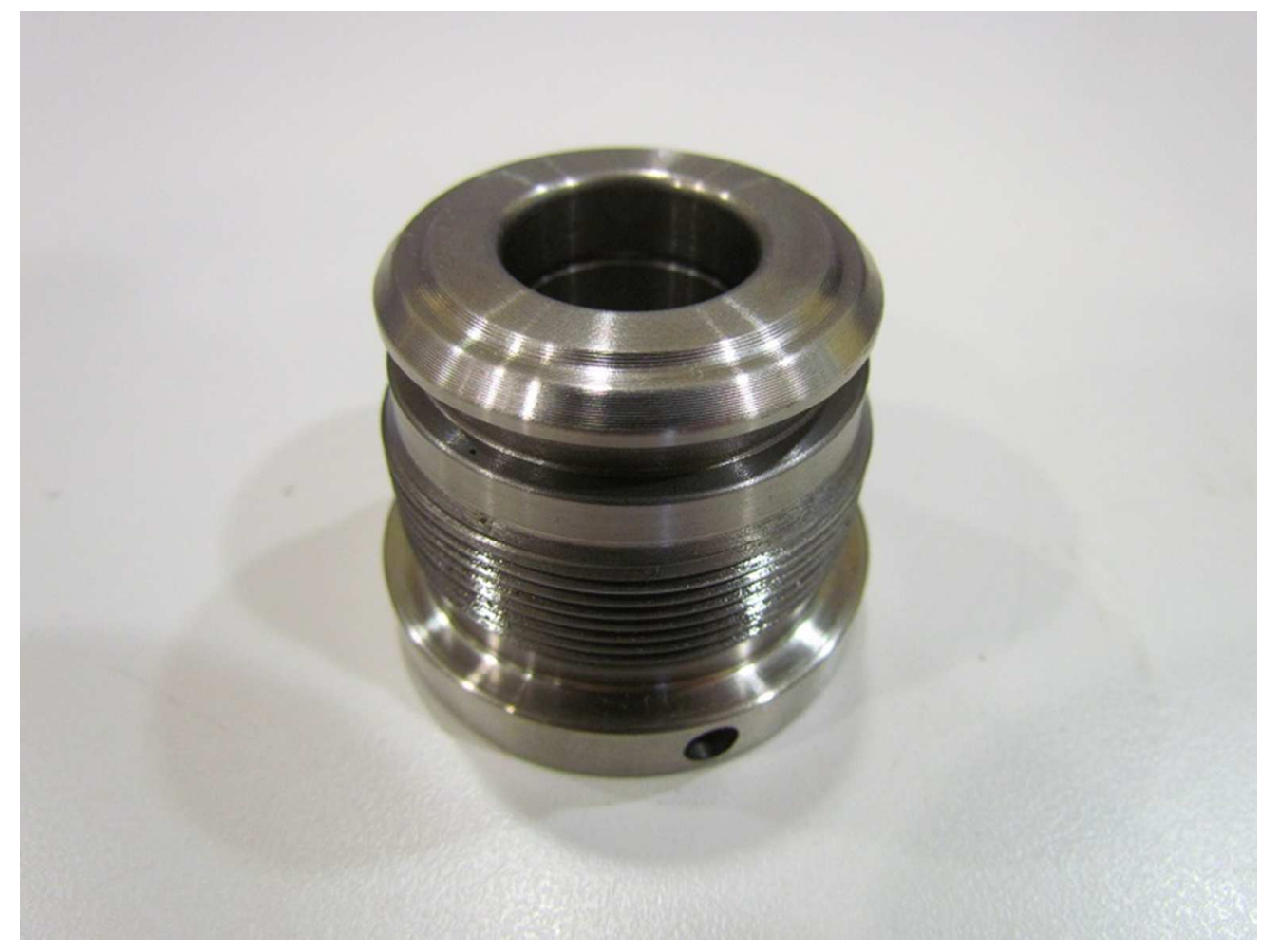

$112 \times 84 \mathrm{~mm}(180 \times 180 \mathrm{DPI})$ 